\title{
Reporting of Prostatic Adenocarcinoma in Needle Biopsies-Gleason's Grading vs Contemporary Group Grading System: Single Centre Experience
}

\author{
Vinay KS and Sujatha Siddappa* \\ Department of Pathology, Institute of Nephro Urology, Victoria Hospital campus, Bangalore, India
}

${ }^{*}$ Corresponding author: Sujatha Siddappa, Department of Pathology, Institute of Nephro Urology, Victoria Hospital campus, Bangalore, India, E-mail: sujathasiddappa@gmail.com

Received: 08 Jan, 2018 | Accepted: 05 Feb, 2018 | Published: 09 Feb, 2018

Citation: Vinay KS, Siddappa S (2018) Reporting of Prostatic Adenocarcinoma in Needle Biopsies-Gleason's Grading vs Contemporary Group Grading System: Single Centre Experience. Clin Res Open Access 4(1): dx.doi.org/10.16966/2469-6714.133

Copyright: (C) 2018 Vinay KS, et al. This is an open-access article distributed under the terms of the Creative Commons Attribution License, which permits unrestricted use, distribution, and reproduction in any medium, provided the original author and source are credited.

\section{Abstract}

Background: Gleason grading system which is used for reporting prostatic adenocarcinoma is an important grading parameter for prognostication and helps in making treatment decisions. Needle biopsy Gleason score correlates well with the final score of majority of radical prostatectomy specimens and even with the serum prostate specific antigen levels. Gleason grading system has evolved from the original scheme summarized by Dr. Donald Gleason in 1970 to a significantly modified system after two major consensus meetings conducted by ISUP in 2005 and 2014 . Original scheme and latest modifications of Gleason grading system which still has some major limitations has led to a new 'Contemporary Grade Group system' proposed by 2014 ISUP which in turn adopted by the 2016 WHO classification of tumors of prostate.

Objective: An observational study to compare traditional Gleason's grading system with contemporary grade grouping system in reporting prostate core biopsies

Materials and Methods: A study of prostatic core biopsies from August 2016 to July 2017 (One year study period) received in the department of pathology, Institute of NephroUrology, Victoria hospital campus, Bangalore, Karnataka, India were included. Biopsies of patients after treatment for prostatic carcinoma and biopsies which did not include any glandular component were not included. Core biopsies (6 cores from each case) were received in $10 \%$ formalin. All the cores were embedded and sections were cut at 3 to 5 microns. Hematoxylin and eosin staining was done and examined by pathologists. Individual cores from each case were screened and reported independently following both 'Gleason grading system' as well as 'Contemporary grade grouping system'. Highest Gleason score/Group grade was mentioned in the final impression.

Results: We received a total number of 46 biopsy samples during the study period. Out of 46 , Seventeen were reported as prostatic adenocarcinoma, 2 were atypical small acinar proliferation (ASAP), 2 were high grade prostatic intraepithelial neoplasia (HGPIN) and the rest were benign prostatic tissue. 17 prostatic adenocarcinoma cases were further classified by both Gleason's / Contemporary grade group system as follows, 2 cases (11. 76\%) - Grade group I [GS 3+3=6/10], 2 cases (11. 76\%) - Grade group II [3+4=7], 7 cases (41. 17\%) - Grade group III [ GS 4+3=7/10], 4 cases (23. 52\%) - Grade group IV [GS $4+4=10$ ], 2 cases(11. 76\%) - Grade group V [ GS 5+4=9/10]. Perineural invasion was seen in 12 out of 17 cases (70. 55\%). Average PSA levels were $37.35 \mathrm{ng} / \mathrm{dL}$. Lymphovascular emboli were not seen in any of the cases.

Conclusion: Gleason system for prostatic cancer is an important and universally (WHO endorsed) used prognostic grading system. It has stood the test of time for more than 4-5 decades. Although modification has been done over the time by consensus conferences, it has got some major limitations. To overcome these limitations, a new contemporary Grade grouping system has been proposed recently. The new Contemporary grade grouping system has simplified 5 grades with lowest possible grade of 1 with more accurate grade stratification. The new grading system has been accepted by the 2016 WHO.

As a pathologist's point of view, reporting of prostate core biopsies based on glandular architecture at low power examination gives better information about the grades. Since we are accustomed to the traditional GS, we should report the Gleason score and also simultaneously concur with the grade of CGGS. We recommend reporting of the prostate biopsies by following both the contemporary grade grouping system along with the traditional Gleason system till it becomes widely accepted and practiced. New grade grouping system is simpler and more precisely represent the prognostication of prostate cancer biology. It is also endorsed by the WHO.

Keywords: Gleason grading system; Contemporary grade grouping system; Prostatic core biopsy; International Society of Urologic Pathology (ISUP); World Health Organization (WHO)

Abbreviations: International Society of Urologic Pathology (ISUP); World Health Organization (WHO) 


\section{Introduction}

Gleason grading system which is used for reporting prostatic adenocarcinoma is an important grading parameter for prognostication and helps in making treatment decisions. Ultrasound guided prostatic core biopsy Gleason score correlates well with the final score of majority of radical prostatectomy specimens and even with the serum prostate specific antigen (PSA) levels [1]. The Gleason grading system was proposed by Dr Donald Gleason based on a study in 1959 [2]. Gleason grading system defined five histological patterns or grades which includes most well differentiated at one end and the least differentiated at the other. The World Health organization (WHO) has included Gleason grading system in 2004 as a part of prostate cancer classification. This system also forms the base for many other cancer staging systems like AJCC/UICC. However many changes have been proposed since its inception to update the original Gleason system. Most important step in this direction is the International Society of Urologic Pathology (ISUP) consensus published in 2005. This meeting brought about a good number of required changes in the existing Gleason system bases on consensus from more than 70 urologic pathologists. Another important meeting was held at Chicago in 2014. It included not only pathologists but also other specialty doctors from urology and oncology who are involved in treatment of patients with prostatic adenocarcinoma [3]. Although many changes were made to the original Gleason system in 2005 and in 2014 meetings, there still exist a lot of shortcomings in its current use for prostatic carcinoma reporting either in prostatic core biopsies or the radical prostatectomy specimens. To address the same, a new grading system was proposed by the group from John Hopkins's hospital. This new prostatic grading system has benefits like more accurate grade stratification than the Gleason grading system [4].

\section{Materials and Methods}

Prostate core biopsies from Aug 2016 to July 2017 received in the department of pathology, Institute of NephroUrology, Bangalore, Karnataka, India were included. Sextant biopsies (6 cores from each case representing left upper, middle and lower lobe and right upper, middle and lower lobe) were received in $10 \%$ formalin. All the cores were embedded and sections were cut at 3-5 microns. Hematoxylin and Eosin (H \& E) staining was done and examined by pathologists. Individual cores from each case were reported independently following both Gleason grading system (GS) as well as Contemporary Grade grouping system (CGGS).

\section{Reporting of Gleason grading system-GS}

GS is based on glandular architecture/histological pattern. GS defines five histological patterns with most well differentiated glands at one end of the spectrum and the least differentiated at the other end. The primary pattern which is most common pattern and the secondary pattern which is the next most prevalent pattern are noted and added together to get a final Gleason score.
- Gleason pattern 1: It includes tumor with glands having mild variation in size and shape and it is well circumscribed. There is no infiltration into surrounding benign prostatic tissue.

- Gleason pattern 2: It includes glands with moderate variation in size and shape with varying amounts of stroma in between. There may be infiltration of tumor glands into the adjacent benign prostatic tissue.

- Gleason pattern 3: It includes proliferation of small glands with moderate variation in size and shape with variable stroma. Infiltration into the adjacent benign prostatic tissue is usually seen.

- Gleason pattern 4: This typically involves small fused glands, both large and small cribriform glands and poorly formed glands. Infiltration into the adjacent benign prostatic tissue is well seen.

- Gleason pattern 5: In this there is no gland formation and the tumor cells are arranged in sheets and in single infiltrative pattern. Infiltration into adjacent benign prostatic tissue is invariably seen.

We reported both primary and secondary pattern and added together to get a Gleason score. Gleason score was provided separately for individual cores. Highest score was mentioned in the final impression.

\section{Reporting the Contemporary Group grading system- CGGS [4]}

CGGS is studied with reference to existing Gleason system for proper understanding and categorization of morphological patterns.

- Grade Group 1: This includes individual discrete well formed glands. This correlates with GS score of $3+3$.

- Grade group 2: It includes predominantly well formed glands with a smaller component of poorly formed / fused / cribriform glands. This correlates with GS score of 3+4.

- Grade group 3: It includes predominantly poorly formed/ fused/cribriform glands with smaller component of well formed glands. This correlates with GS score of $4+3$.

- Grade group 4: It includes only the poorly formed / fused/cribriform glands. This correlates with GS score $4+4 / 3+5 / 5+3$. It can also include predominantly well formed glands with a smaller component of lacking glands i.e., GS score of $3+5$. It can also include predominantly loss of gland formation and a smaller component of well formed glands i.e., GS score of $5+3$.

- Gleason pattern 5: It is the poorly differentiated with total loss of gland formation (or with necrosis) with or without a component of poorly formed/fused/cribriform glands.

If the cases with $>95 \%$ poorly formed/fused/cribriform glands or loss of glands on a core, the component of $<5 \%$ well formed gland is not included for grading Poorly formed/fused/ cribriform glands can also be a part of minor component. 
CGGS was reported for individual cores with highest grade mentioned in the final impression.

We mentioned both the GS score and CGGS in the reports. Other histopathological parameters which are not included in both the grading system like perineural invasion (PNI), lymphovascular emboli (LVE) were mentioned and included in the histopathology report.

Associated features like atypical small acinar cell proliferation (ASAP) and high grade prostatic intraepithelial neoplasia (HGPIN) were noted and mentioned in the histopathology report when found in particular cases (Figure 1-4).

\section{Results}

We received a total of 46 core biopsy samples during the study period. Out of 46 , Seventeen were reported as prostatic adenocarcinoma, 2 were ASAP, 2 were HGPIN and rest were benign prostatic tissue.
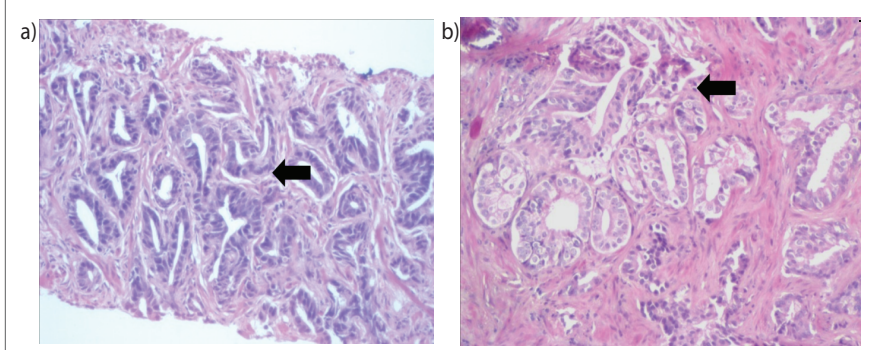

Figure 1a \& 1b: Gleason pattern 3: Glands with marked variation in size and shape lined by cells having hyperchromatic nuclei with scanty cytoplasm. Basal cells are not seen. CGGS 1.

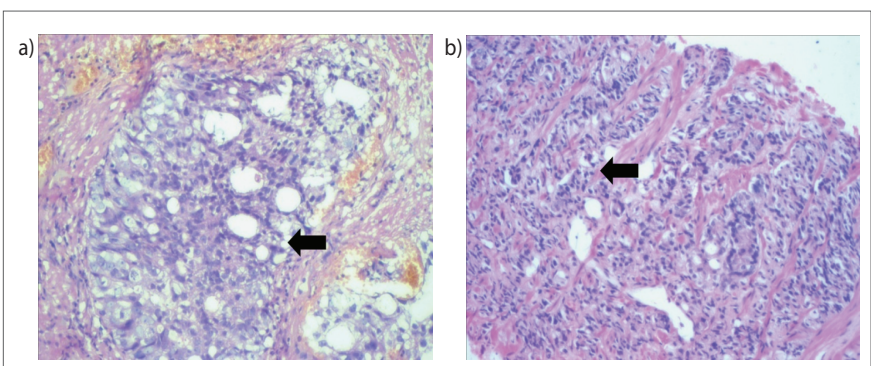

Figure 2a \& 2b: Gleason pattern 4: Poorly formed fused prostatic glands with cribriform pattern and fused glands with poorly formed lumina CGGS $2 \& 3$.

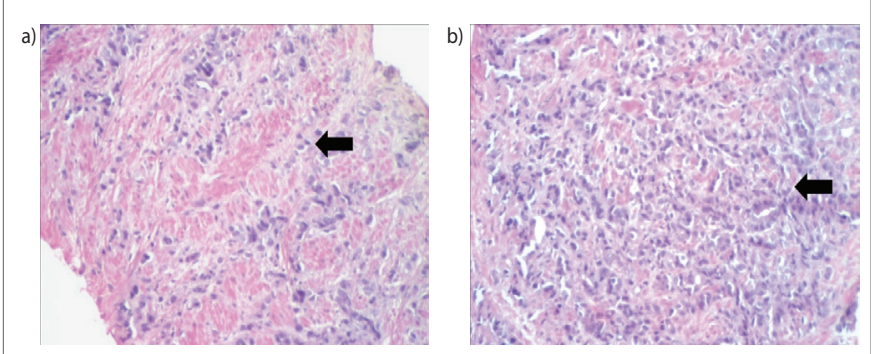

Figure 3 (a) \& (b) - Gleason pattern 5: Tumor cells in single cell infiltrative pattern and in sheets with attempted vague gland formation CGGS 4 \& 5 .

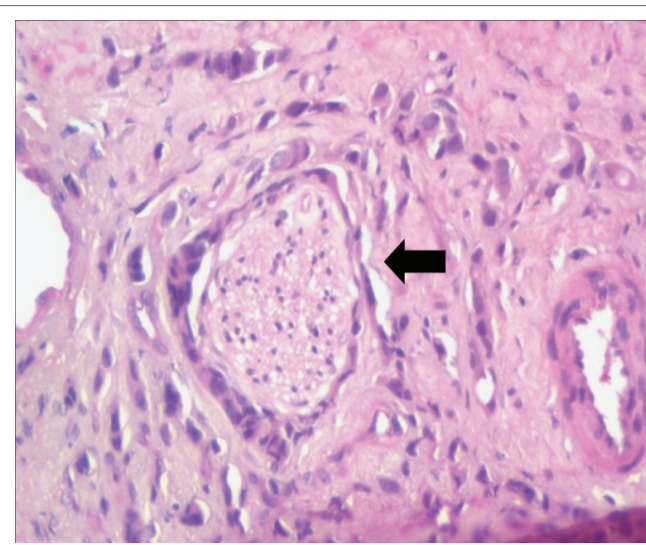

Figure 4: Perineural invasion: Nerve fiber with perineural tumor invasion.

17 prostatic adenocarcinoma cases were further classified by both Gleason's / Grade group system as follows

2 cases $(11.76 \%)$ - Grade group I (GS 3+3=6)

2 cases (11. 76\%) - Grade group II (GS $3+4=7 / 10$ )

7 cases $(41.17 \%)$ - Grade group III (GS $4+3=7 / 10$ )

4 cases (23. 52\%) - Grade group IV (GS $4+4=8$ )

2 cases (11. 76\%) - Grade group V (GS 5+5/5+4/4+5=9-10)

PNI was seen in 12 out of 17 cases (70.55\%)

PSA values were available in 29 cases. Average PSA levels were $37.35 \mathrm{ng} / \mathrm{dL}$. LVE was not seen.

\section{Discussion}

GS till date is very important predictor of prognosis in prostatic adenocarcinoma and helps surgeons and oncologists to decide the clinical management. GS differ from other tumor classification that it is based on glandular pattern or the architecture rather than tumor cell morphology and it is used worldwide [5]. Although 2005 and 2014 ISUP grading consensus conferences have made significant changes for the original GS [6-9], still there are some major limitations. The Most important problems with the current Gleason system is,

- Scores 2 to 5 are no longer used for reporting now since minimum score of 6 is the lowest possible score. Previously reported score of 6 are now graded as 7 after the consensus meeting, thus making GS score 6 cancers having a better prognosis than previous score 6 cancers.

- For any research studies or for prognostic or therapeutic purposes Gleason scores are usually divided into 3 groups i.e., GS 6, 7 and 8-10. For cases with GS score of 7, which can be as a result of $4+3$ or $3+4$, both have a different prognosis i.e., GS $3+4=7$ having better prognosis than $4+3=7$.

- In routine histopathology reporting of prostatic adenocarcinomas, the lowest possible score is 6 , although the theoretical possible score is 2 . So it will be assumed by patient part that the diagnosed cancer with GS score of 6 is almost crossing the middle of the cancer scale $2-10$. This 
leads to the fear and psychological stress that the cancer is serious and the treatment is mandatory, whereas majority of the GS score 6 cancers behave indolently without the much needed ancillary therapy apart from surgery.

To come up with the above shortcomings new CGGS is proposed. This system is based on the data from the John Hopkins hospital which included $>20,000$ patients treated by surgery and $>5000$ patients treated by radiotherapy. This was validated in a multi centric and multi institutional study. This new CGGS includes only five distinct grade group based on modified Gleason score groups as mentioned before in the study format. This new grading system has been accepted and included as a part of prostatic cancer classification by the World Health Organization in 2016. This study has taken biochemical recurrence as the endpoint for prognostication of different grades [4]. This reporting system can be further optimized by incorporating the additional histological factors like reactive stroma into routine assessment [10]. In our observational study, the study period is 1 year and there was no correlation with radical prostatectomies. For prognostic purposes cancer recurrence has to be followed up over long periods with biochemical recurrence which is not done in our study. There is limited number of pathological research studies comparing the traditional $v s$ contemporary grade grouping system.

\section{Conclusion}

Gleason system for prostatic carcinoma is an important and universally used grading parameter for prognostication and classifying patients based on glandular architecture. It has stood the test of time for more than 4-5 decades. Although modification has been done over the time by consensus conferences, it has got some major limitations. To overcome these limitations, a new contemporary Grade grouping system has been proposed recently. The new Contemporary grading system has simplified 5 grades with lowest possible grade of 1 with more accurate grade stratification. The new grading system has been accepted and included in prostate cancer classification by world health organization in 2016.

As a pathologist's point of view, reporting of prostate core biopsies based on glandular architecture at low power examination gives better information about the grades. Since we are accustomed to the traditional GS, we should report the score and also simultaneously concur with the grade of CGGS. It may be difficult initially to follow the CGGS without the scoring of GS. We recommend reporting by following contemporary grade grouping system (CGGS) along with the traditional Gleason system (GS) till it becomes widely accepted and practiced. We also feel perineural invasion, lymphovascular emboli and any associated HGPIN although not included in any of the grading systems, and they should be mentioned in the final impression so that it may help with the treating urologists in making treatment decisions.

\section{References}

1. Humphrey PA (2007) Diagnosis of adenocarcinoma in prostate needle biopsy tissue. J Clin Pathol 60: 35-42.

2. Gleason DF, Mellinger GT (1974) Prediction of prognosis for prostatic adenocarcinoma by combined histological grading and clinical staging. J Urol 111: 58-64.

3. Ni Chen, Quao Zhou (2016) The evolving Gleason grading system. Clinical J Cancer Res 28: 58-64.

4. Epstein JI, Zelefsky MJ, Sjoberg DD, Nelson JB, Egevad L, et al. (2016) A Contemporary Prostate Cancer Grading System: A Validated Alternative to the Gleason Score. Eur Urol 69: 428-435.

5. Gordetsky J, Epstein JI (2016) Grading of prostatic adenocarcinoma: current state and prognostic implications. Diagn Pathol 11: 25.

6. Epstein JI, Allsbrook WC Jr, Amin MB, Egevad LL, ISUP Grading Committee (2005) The 2005 International Society of Urological Pathology (ISUP) Consensus Conference on Gleason Grading of Prostatic Carcinoma. Am J Surg Pathol 2005 29: 1228-1242.

7. Epstein JI, Egevad L, Amin MB, Delahunt B, Srigley JR, et al. (2016) The 2014 International Society of Urological Pathology (ISUP) Consensus Conference on Gleason Grading of Prostatic Carcinoma: Definition of Grading Patterns and Proposal for a New Grading System. Am J Surg Pathol 40: 244-252.

8. Shah RB, Zhou M (2016) Recent advances in prostate cancer pathology: Gleason grading and beyond. Pathol Int 66: 260-272.

9. Epstein JI, Amin MB, Reuter VE, Humphrey PA (2017) Contemporary Gleason Grading of Prostatic Carcinoma: An Update With Discussion on Practical Issues to Implement the 2014 International Society of Urological Pathology (ISUP) Consensus Conference on Gleason Grading of Prostatic Carcinoma. Am J Surg Pathol 41: e1-e7.

10. McKenney JK, Wei W, Hawley S, Auman H, Newcomb LF, et al. (2016) Histologic Grading of Prostatic Adenocarcinoma Can Be Further Optimized: Analysis of the Relative Prognostic Strength of Individual Architectural Patterns in 1275 Patients From the Canary Retrospective Cohort. Am J Surg Pathol 40: 1439-1456. 